(2) Open Access Full Text Article

\title{
Impacts of age and gender at the risk of underlying medical conditions and death in patients with avian influenza $A$ (H7N9): a meta-analysis study
}

This article was published in the following Dove Press journal:

Therapeutics and Clinical Risk Management

\author{
Qinglin Cheng ${ }^{1,2, *}$ \\ Gang Zhao',* \\ Li Xie ${ }^{1, *}$ \\ Xuchu Wangl,*
}

'Division of Infectious Diseases, Hangzhou Center for Disease Control and Prevention, Hangzhou 31002I, China; ${ }^{2}$ Department of Adolescents and Children Health, School of Public Health, Medical College of Soochow University, Suzhou 215123, China

*These authors contributed equally to this work
Correspondence: Xuchu Wang Division of Infectious Diseases, Hangzhou Center for Disease Control and Prevention, 568 Mingshi Road, Hangzhou 31002I, China

Tel/fax +86 57l 88000505

Email wanghzcdc@sina.com
Objective: The objective of our study was to conduct a series of analyses that examined the impacts of age and gender at the risk of underlying medical conditions (UMCs) and death in patients with influenza A (H7N9).

Methods: We began by searching for potentially relevant articles in English or Chinese before February 28, 2018. Additionally, we reviewed our own files and reference lists of articles identified by this search.

Results: The association between death and UMCs was significant in H7N9 patients, with an OR of 1.49 (95\% CI: 1.24-1.78). Subgroup analyses showed that having two or more UMCs of any type (OR: $2.24 ; P=0.044)$, chronic respiratory diseases (OR: $1.81 ; P=0.032$ ), and chronic cardiovascular disease (OR: $1.63 ; P=0.013)$ had an association with increased fatality in H7N9 patients. Age ( 60 years or older) [adjusted OR (AOR): $1.86 ; P=0.032$ ] and gender (male: AOR: 1.68, $P=0.006$; female: AOR: $1.88, P=0.044$ ) were significantly associated with death in H7N9 patients with UMCs compared to H7N9 patients without any UMC. Stratification analyses found statistically significant increased death in H7N9 patients with UMCs who were 60 years of age and older (AOR: 2.72; $P<0.001$ ) and gender (male; AOR=1.64; $P=0.033$ ), compared to H7N9 patients without these respective conditions.

Conclusion: Impacts of age are substantial and significant at the risk of UMCs and death in H7N9 patients. This analysis did not find a significant difference in gender comparisons. Efforts should particularly focus on reducing fatality rates in patients with combined risks from UMCs and other significant impact factor such as age (60 years or older).

Keywords: avian influenza virus, H7N9, fatality, underlying medical conditions, meta-analysis

\section{Introduction}

Emerging and re-emerging infectious diseases constitute a continuing threat to human health. Among them, the influenza A (H7N9) virus causes severe disease in humans and poses an unprecedented pandemic threat. By February 28, 2018, there had been 1,567 laboratory-confirmed human H7N9-infected cases across the world, and severe illness and case-fatality risk from H7N9 have been recorded in many research reports. ${ }^{1,2}$ In reviewing the literature, we found that young children typically presented with only mild infections, while older adults presented with more severe symptoms. ${ }^{2}$ Although previous studies have shown that H7N9 infection is often accompanied by multiple chronic conditions (hypertension, diabetes, chronic bronchitis, etc.), ${ }^{3-6}$ the most recent H7N9 epidemic seems to reveal a different increased risk of death in 
elderly patients with particular chronic underlying medical conditions (UMCs). ${ }^{3,6,7}$

A major point of discussion in the field of infectious disease control is whether UMCs are an independent risk factor for worsened H7N9 outcomes, or whether the association between UMCs and worsened H7N9 outcomes is the result of noncausal mechanisms. ${ }^{5-8}$ Previous studies were only able to provide estimates of unadjusted associations, or very limited estimates of adjusted associations, ${ }^{4-8}$ because the wide variability of adjustments in individual studies makes comparisons across studies very difficult. It is nonetheless evident that patients with more medical risk factors are at higher risk of adverse H7N9 outcomes, such as the acute respiratory distress syndrome and death. ${ }^{6,7}$ Most importantly, some potential impact factors (such as age and gender) ${ }^{4-8}$ may confound the association between UMCs and H7N9 infection outcome, as exposure to more risk factors could result in both a higher fatality from H7N9 infection as well as worsened prognosis. Investigating the effects of potential confounding variables is particularly important because some variables related to potential impact factors are prognostic for the risk of death in H7N9-infected cases, and are also associated with UMCs.

Appropriate public health responses to infectious disease threats should be based on the best available evidence, which in turn requires reliable data and appropriate analysis. In particular, risk assessment for H7N9 involves estimation and characterization of transmissibility and clinical severity. Numerous studies have shown that meta-analyses can provide important insights into the epidemiology and clinical application of a specific disease. The objective of our study was to conduct meta-analyses that examined the impacts of age and gender at the risk of UMCs and death in H7N9 patients. Our results can inform the public health response and help reduce fatality rates.

\section{Materials and methods}

\section{Search strategy}

On February 28, 2018, we extracted articles with estimates of fatality risk of H7N9-infected cases from nine databases (including Ovid-Medline, PubMed, BioMed Central, BMJ, Elsevier ScienceDirect, SpringerLink, Wiley InterScience, China Academic Journal Network Publishing Database [CAJD], and Wanfang Data). The following free search terms were used to search in "All Fields": 1. fatalit* or mortality or death or lethalit*; 2. chronic disease* or underlying medical condition* or chronic underlying medical condition* or chronic medical condition* or chronic condition* or underlying condition* or underlying disease*; 3. \#1 and \#2; 4. influenza or flu; 5. pandemic; 6 . \#4 and \#5; 7. H7N9; 8. \#6 or \#7; 9. \#3 and \#8. No time limitation was set for the searches.

All titles identified via the search strategy were independently screened by two authors. Abstracts of potentially relevant articles and the full texts of manuscripts were reviewed for eligibility. Articles in all languages were selected for assessment if at least one statistical analysis of the association between UMCs and deaths was presented and described as an estimate for H7N9 outcome. We manually retrieved any additional relevant studies identified.

\section{Inclusion/exclusion criteria}

All case-control and cohort studies that measured the association between UMCs and fatality rates for H7N9 were considered for inclusion. Studies were ultimately selected if they (a) were published in English or Chinese; (b) included H7N9-infected patients; (c) reported H7N9-related UMCs as a predictor of clinical outcomes (recovered or died), including chronic cardiovascular diseases (CCD), chronic metabolic diseases, chronic respiratory diseases (CRD), chronic liver disease, coronary heart disease, and rheumatic diseases; and (d) reported any one of the following statistics: case-fatality ratio and sample sizes, or other statistics that could be converted into a standardized effect size. Studies were excluded if (a) no primary data were reported; (b) full-text articles were not available after exhaustive searches to locate them; (c) there were insufficient or missing data (after contacting authors); or (d) groups of studies had considerable heterogeneity (variation in outcomes between studies). Studies with rare UMCs as a predictor of clinical outcomes such as schistosomiasis, articular gout, and benign prostatic hyperplasia were also excluded as these outcomes were unique to one study and, therefore, not meta-analyzable.

\section{Data extraction process}

All data were extracted independently and entered onto a standardized form by two of the authors. The primary data extracted were estimates of fatalities. Whenever available, we extracted fatalities data stratified by age, gender, UMC type, and the proportion of cases with $\geq 2$ UMCs. We also contacted individual authors for the above factors' breakdowns in H7N9 cases and deaths if this information was not reported in the published paper. One person undertook the initial selection of studies, and another reviewed the selection. 
Disagreements were resolved by discussion and consultation with a third author until a consensus was reached.

\section{Quality appraisal}

The quality appraisal tool was adapted from the 9-star Newcastle-Ottawa Scale (NOS) ${ }^{9}$ for all study designs. A full score is nine stars, and a score $\geq 6$ stars is considered to be fair. The NOS contains eight items, separated into three categories: selection, comparability, and outcome (cohort studies) or exposure (case-control studies). Quality assessments for included studies were completed by two independent reviewers.

\section{Statistical methods}

The effect size statistic was defined as the association between death and UMCs in H7N9 patients. Statistical data such as OR or adjusted OR (AOR) [with 95\% CIs] and casefatality ratios were recorded. Our meta-analysis examined the association between death and UMCs in H7N9 patients for each study by OR or AOR with $95 \%$ CI. Summary effect sizes were calculated with inverse variance weighting. All statistical analyses were conducted using Stata 14.0 (StataCorp LP, College Station, TX, USA). Statistical significance was set at $P<0.05$. All statistical tests were two-sided.

Statistical heterogeneity was evaluated through the Q test and $I^{2}$ statistic; $P<0.05$ was considered statistically significant. If the heterogeneity was acceptable $\left(I^{2}<50 \%\right)$, a fixed-effects analysis was conducted to calculate the pooled OR or AOR. Otherwise, a random-effects model was used. To evaluate whether publication bias might affect the statistical results, we applied funnel plots, Egger's test, and Begg's method to assess bias. We also used the so-called leave-oneout function ${ }^{10}$ for doing sensitivity analyses. This method consists of the removal of one study at a time from the dataset to run the meta-analysis without it. This analysis tests if the effect size of the meta-analysis is driven by one study. The method should not be regarded as a way of yielding a more valid estimate of the overall effect or outcome.

\section{Results}

\section{Identification, screening, and eligibility}

Figure 1 illustrates the study inclusion/exclusion process. The full texts of all 146 articles were checked against the general and specific inclusion criteria. This led to the exclusion of 131 articles so that a total of 15 studies $(2.21 \%$ of all titles and abstracts) were included in the final series of meta-analyses via formal searches. ${ }^{4-7,11-21}$

\section{Description of included studies}

Fifteen studies were included in our meta-analysis (see Table 1 for the characteristics of the included studies). The total sample size was 1,767 (including a possible overlapping across part of the patient samples used). All studies were conducted in China. Among these 15 studies, 14 were retrospective case-control studies (NOS scores: one article was five stars and 13 articles were six stars and above, respectively), ${ }^{4-7,11,12,14-21}$ and one was a cohort study (NOS score: seven stars). ${ }^{13}$

\section{Impacts of age and gender on the association between the risk of UMCs and the death of H7N9-infected patients}

A fixed-effects model was used for the comparison between the odds of death among those with UMCs and those without UMCs, ${ }^{4-7,11,12,14-21}$ because an $I^{2}$ value of $0.0 \%$ was found when the data were fitted to a fixed-effects model. The pooled summary estimates from these studies indicated that UMCs significantly increased the risk of death in H7N9 patients (OR: 1.49 ; 95\% CI: $1.24-1.78, z=4.32 ; P<0.001$ ), with no heterogeneity among studies $\left(\mathrm{Q}=7.61, P=0.909, I^{2}=0.0 \%\right)$ (Figure 2).

We designed a subgroup meta-analysis to explore the association between UMC types and death in H7N9 patients. In this subgroup analysis, in which we analyzed the presence or absence of certain UMC types, we found that having two or more UMCs (OR: 2.24; 95\% CI: 1.02-4.92, $z=2.01$; $P=0.044),{ }^{12-14,17} \mathrm{CRD}$ (OR: 1.81; 95\% CI: 1.05-3.11, $z=2.15$; $P=0.032),{ }^{4,5,7,12,14,19,21}$ and CCD (OR: 1.63 ; 95\% CI: $1.11-2.38$, $z=2.49 ; P=0.013)^{4-7,13,14,19,21}$ had a statistically significant association with death. No associations were found for other UMC types $(P>0.05)$ (Figure 2$)$.

We also conducted a subgroup meta-analysis on UMCs associated with fatalities of H7N9-infected cases by adjusting for the effects of age and gender. As shown in Figure 2, we found that age (60 years or older; AOR: 1.86; 95\% CI: 1.05-3.29, $z=2.14 ; P=0.032)^{6,13,15-17,19,21}$ and gender [male: AOR: 1.68 (95\% CI: 1.16-2.45), $z=2.73$, $P=0.006$; female: AOR: 1.88 (95\% CI: 1.03-3.59), $z=1.99$, $P=0.044]^{5,13,15-17,19,21}$ were significantly associated with death in H7N9 patients with UMCs compared to H7N9 patients without any UMCs. However, differences in 0-59 years aged subgroup were not significantly associated with changes in the risk of death regardless of UMCs $\left(P>0.05, I^{2}<50.0 \%\right)$ (Figure 2). ${ }^{6,13,15-17,19,21}$ 


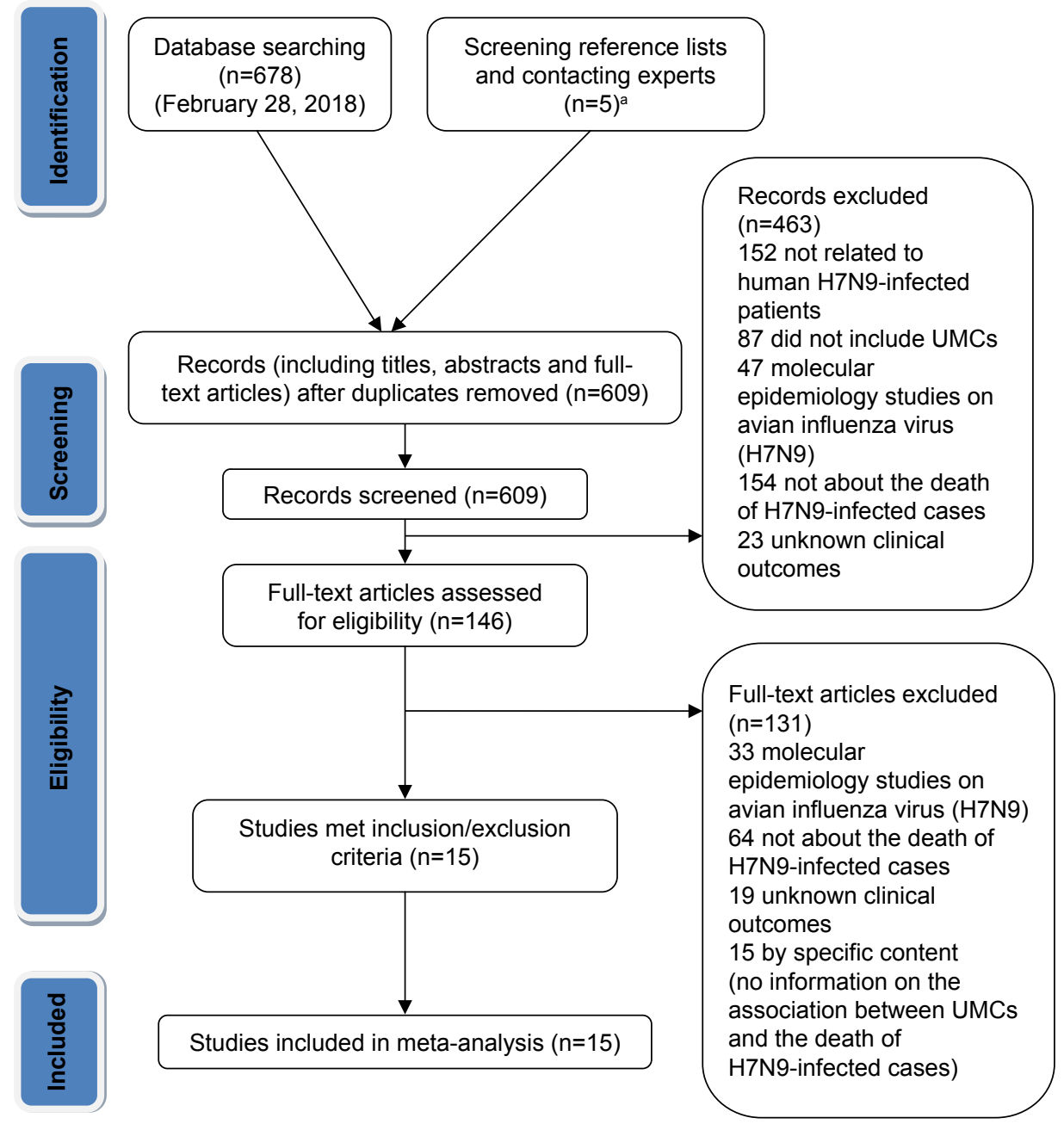

Figure I Flow chart of selection of publications for inclusion in meta-analysis. Note: ${ }^{a}$ Five authors were contacted for additional data to determine eligibility. Abbreviation: UMCs, underlying medical conditions.

Stratification comparisons were also conducted to examine the combined effects of UMCs with age or gender on fatality rates among H7N9 patients. Statistically significant increased fatality rates were observed in $\mathrm{H} 7 \mathrm{~N} 9$ patients with UMCs who were 60 years and older (AOR: 2.72; 95\% CI: $1.72-4.28, z=4.31 ; P<0.001)$, compared to H7N9 patients with UMCs who were $0-59$ years old. ${ }^{6,13-17,21}$ This analysis did not find a significant difference in gender comparisons $\left(P>0.05, I^{2}<50.0 \%\right.$ ) (Figure 3)..$^{5,13-17,21}$

\section{Assessments of heterogeneity and publication bias}

For each meta-analysis, Table 2 shows the summary Q test of homogeneity, the heterogeneity variance between studies (measured by $I^{2}$ statistics), and publication bias assessments including Egger's test and Begg's method. The $\mathrm{Q}$ test and $I^{2}$ statistics indicated that there was no evidence of heterogeneity among studies included into final models (Table 2).

Funnel plots presented the association between log OR and standard error based on the results of meta-analyses, and each point represents a study (Figure 4). There was generally no evidence of publication bias among the included observational studies (Table 2, Figure 4).

\section{Sensitivity analysis}

The sensitivity analysis indicated that the omission of any of the studies led to changes in estimates: (1) the pooled estimate of the risk of UMCs and death in H7N9 patients was between OR: 1.32 (95\% CI: 1.05-1.66) and OR: 1.57 (95\% CI: 1.29-1.93); (2) the estimates by adjusting for the effects of age and gender: 1) the estimate by adjusting for the effect of 60 years of age and older was between OR: 1.71 (95\% CI: 1.02-3.17) and OR: 2.02 (95\% CI: 1.03-3.95); 


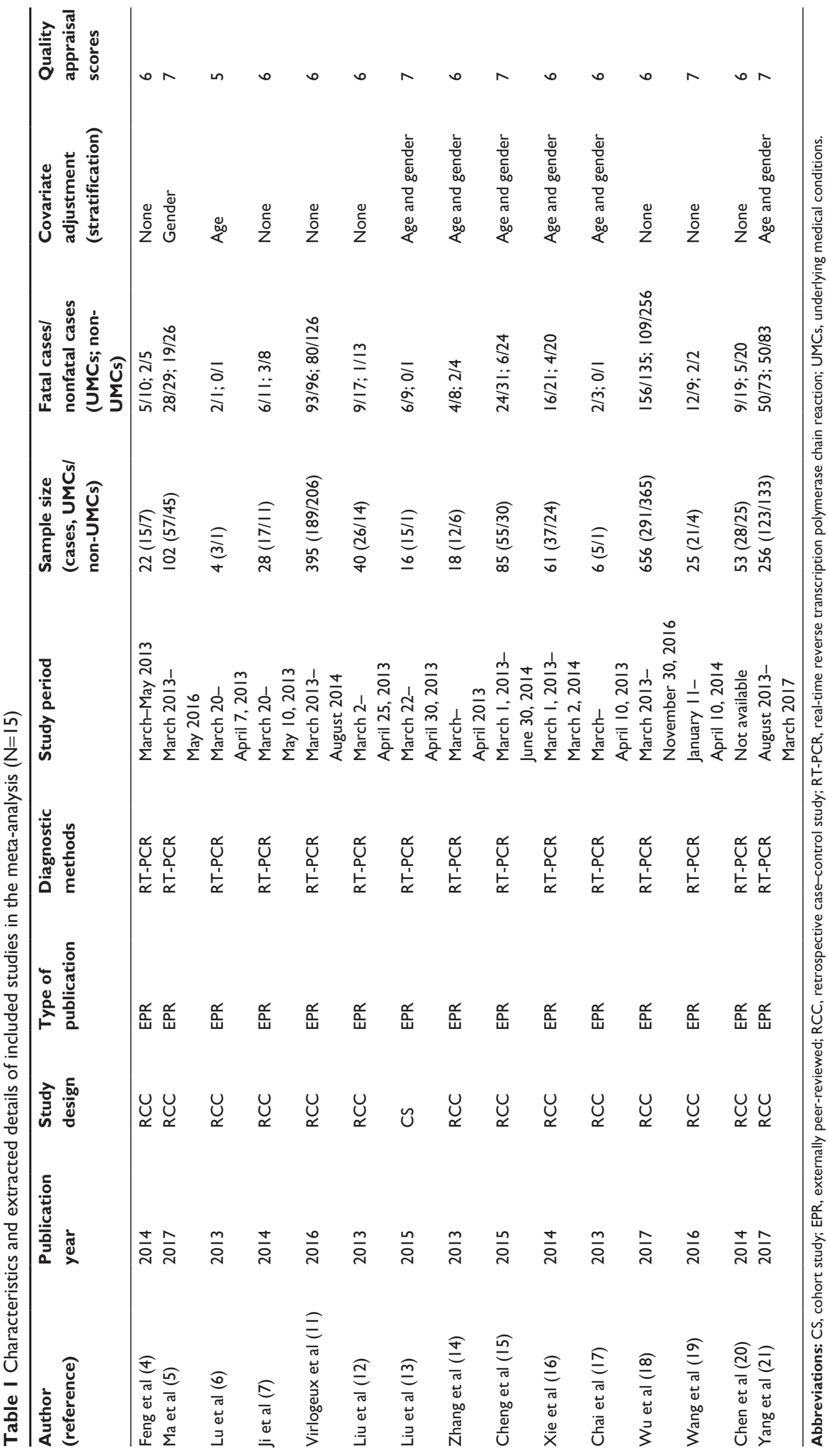


2) the estimate by adjusting for the effect of 0-59 years old was between OR: 1.20 (95\% CI: 0.52-2.74) and OR: 2.23 (95\% CI: 1.00-4.97); 3) the estimate by adjusting for the effect of male was between OR: 1.53 (95\% CI: 1.01-2.42) and OR: 1.76 (95\% CI: 1.15-2.69); 4) the estimate by adjusting for the effect of female was between OR: $1.63(95 \%$ CI: 1.00-3.29) and OR: 2.03 (95\% CI: 1.25-4.35); (3) the estimate of combined effects of UMCs with age (60 years of age and older vs 0-59 years old) was between OR: $1.79(95 \%$ CI: 1.00-3.37) and OR: 2.99 (95\% CI: 1.77-5.06); (4) the estimate of combined effects of UMCs with gender (male vs female) was between OR: 1.40 (95\% CI: 0.86-2.29) and OR: 1.82 (95\% CI: 1.09-3.03). Sensitivity analysis of effect size in statistical models showed no significant differences for the included observational studies (Figure 5).

\section{Discussion}

Our analysis found that UMCs were significantly associated with increased fatality rates among H7N9-infected cases. Similarly, Yang et al analyzed the data of H7N9 case fatalities in Guangdong Province identified as of March 2017, and also showed that $50.0 \%(50 / 100)$ of dead patients infected with H7N9 had a history of exposure to UMCs. ${ }^{21}$ However, our findings suggest that not all UMCs are associated with increased fatality rates in H7N9 patients. In our subgroup analyses, we found that only having two or more UMCs, CRD, and CCD were associated with increased risk of death in H7N9 patients. Similar associations were also reported in the 2009 influenza A H1N1 infection. ${ }^{22-24}$ Consistent with our results, $\mathrm{Wu}$ et $\mathrm{al}^{18}$ described a pattern of increased fatalities in H1N1 patients with multiple UMCs. Ma et al used the data obtained from March 1, 2013 to June 1, 2016 in Jiangsu Province and found significant differences in the CRD and CCD between fatal and nonfatal H7N9 cases. $^{5}$ Our findings suggest that early diagnosis and treatment of CRD, CCD, and multiple comorbid UMCs may more effectively reduce fatalities and disease burden among the H7N9-infected patients.

To our knowledge, ours is the first comprehensive analysis of the adjusted association between UMCs and death in $\mathrm{H} 7 \mathrm{~N} 9$ patients. It is also known that multiple UMCs are often related to old age, and the increased fatality rate among both $\mathrm{H} 1 \mathrm{~N} 1$ and H7N9 patients with multiple comorbid UMCs may be due to decreased immune function related to old age as well as/or the presence of UMCs. ${ }^{25-27}$ It is worth highlighting that our subgroup analyses showed

\section{A Study ID}

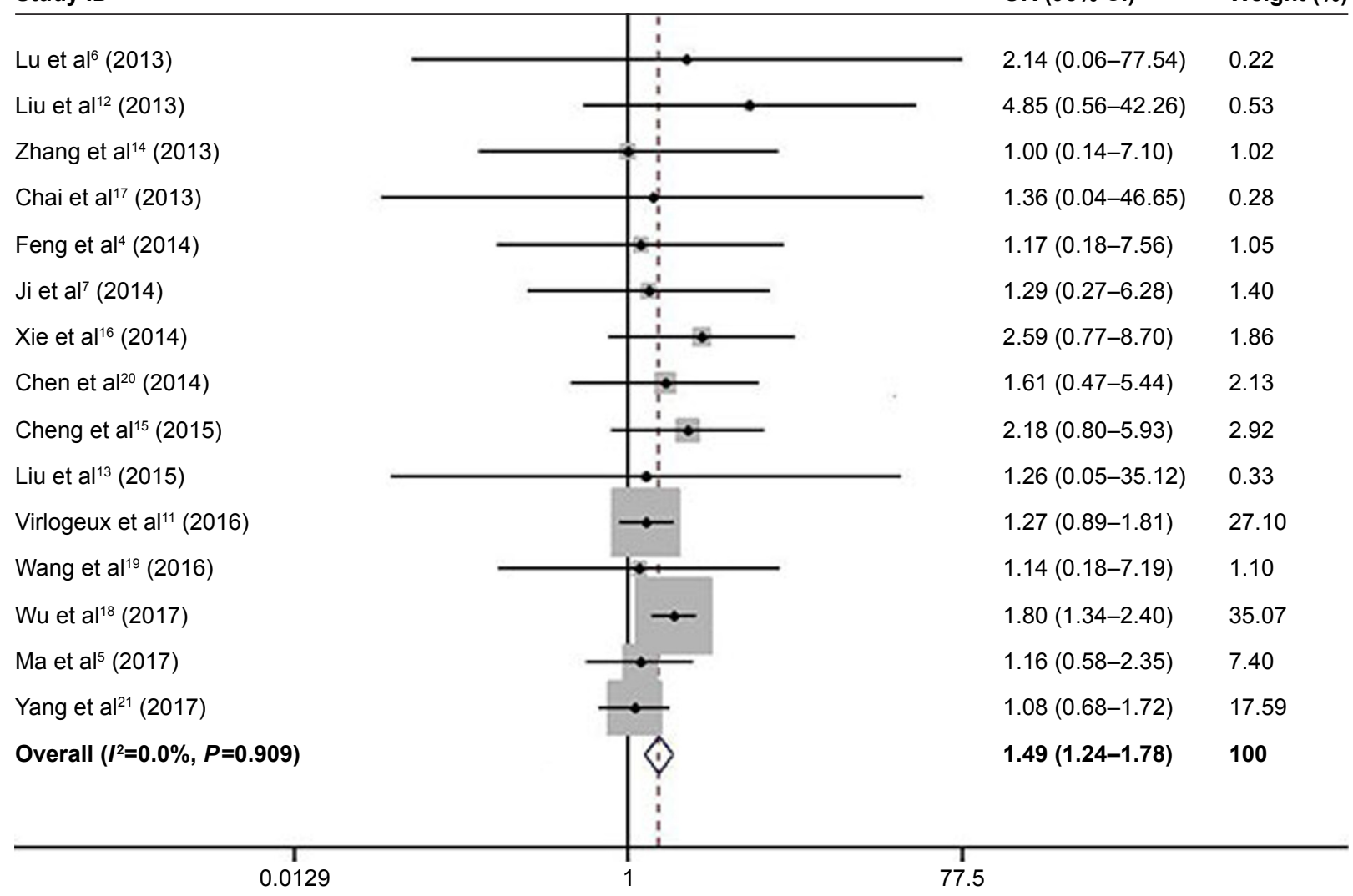

Figure 2 (Continued) 


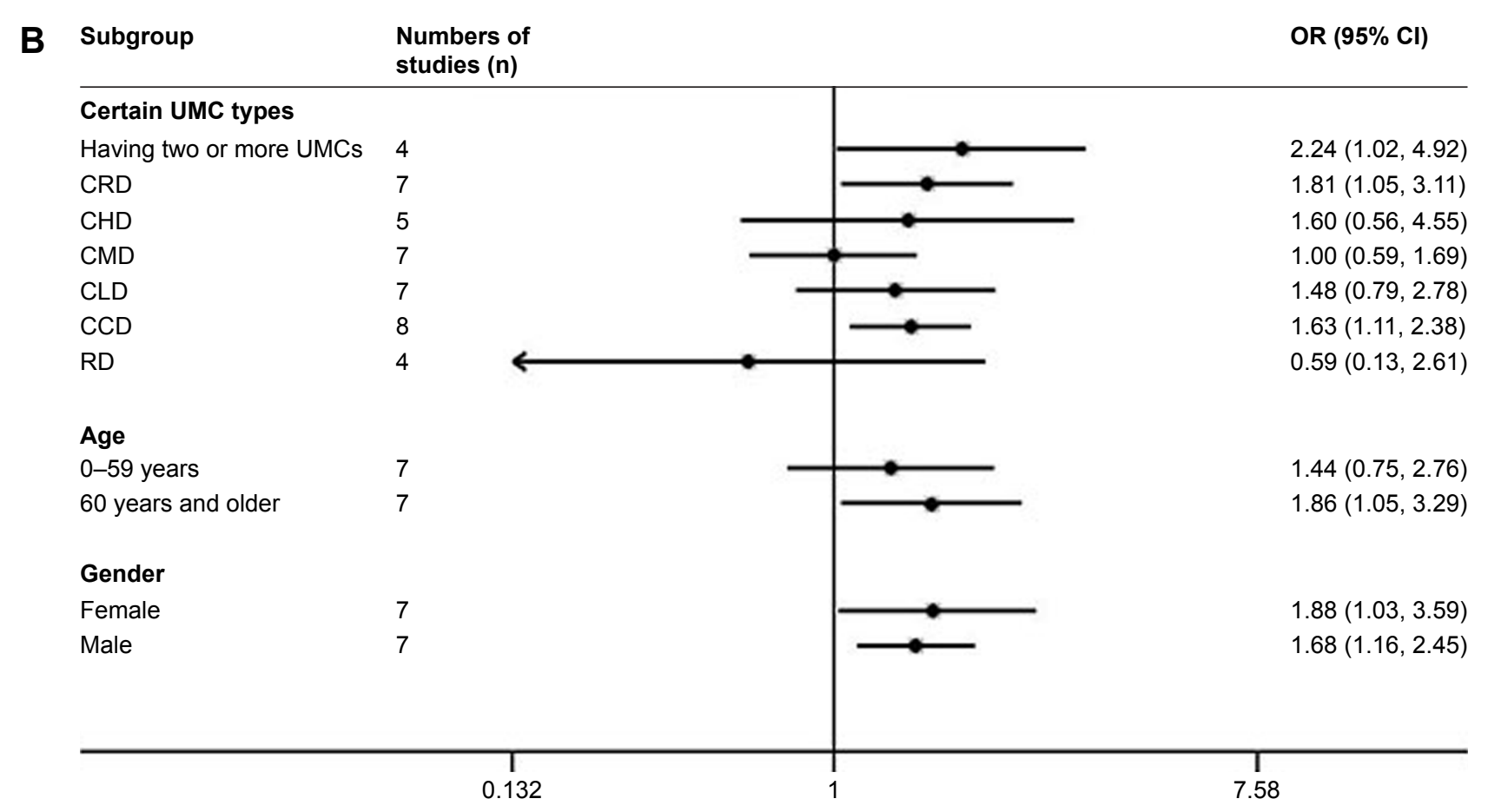

Figure 2 Forest plots on effect sizes of underlying medical conditions associated with fatalities of H7N9-infected cases.

Notes: (A) The pooled estimate. (B) Subgroup analyses of certain UMC types, age, and gender.

Abbreviations: CCD, chronic cardiovascular diseases; CHD, coronary heart disease; CLD, chronic liver disease; CMD, chronic metabolic diseases; CRD, chronic respiratory diseases; RD, rheumatic diseases; UMC, underlying medical condition.

that UMCs were significantly associated with increased fatality rates in H7N9 patients even after adjusting for age and gender. Previous studies have found that age and UMCs were the only independent factors associated with death in
H7N9 patients. ${ }^{5,12,19,28}$ Our findings suggest that preexisting UMCs are only associated with increased fatality rates among older H7N9 patients. By contrast, we found no evidence that UMCs affect the risk of death in H7N9 patients

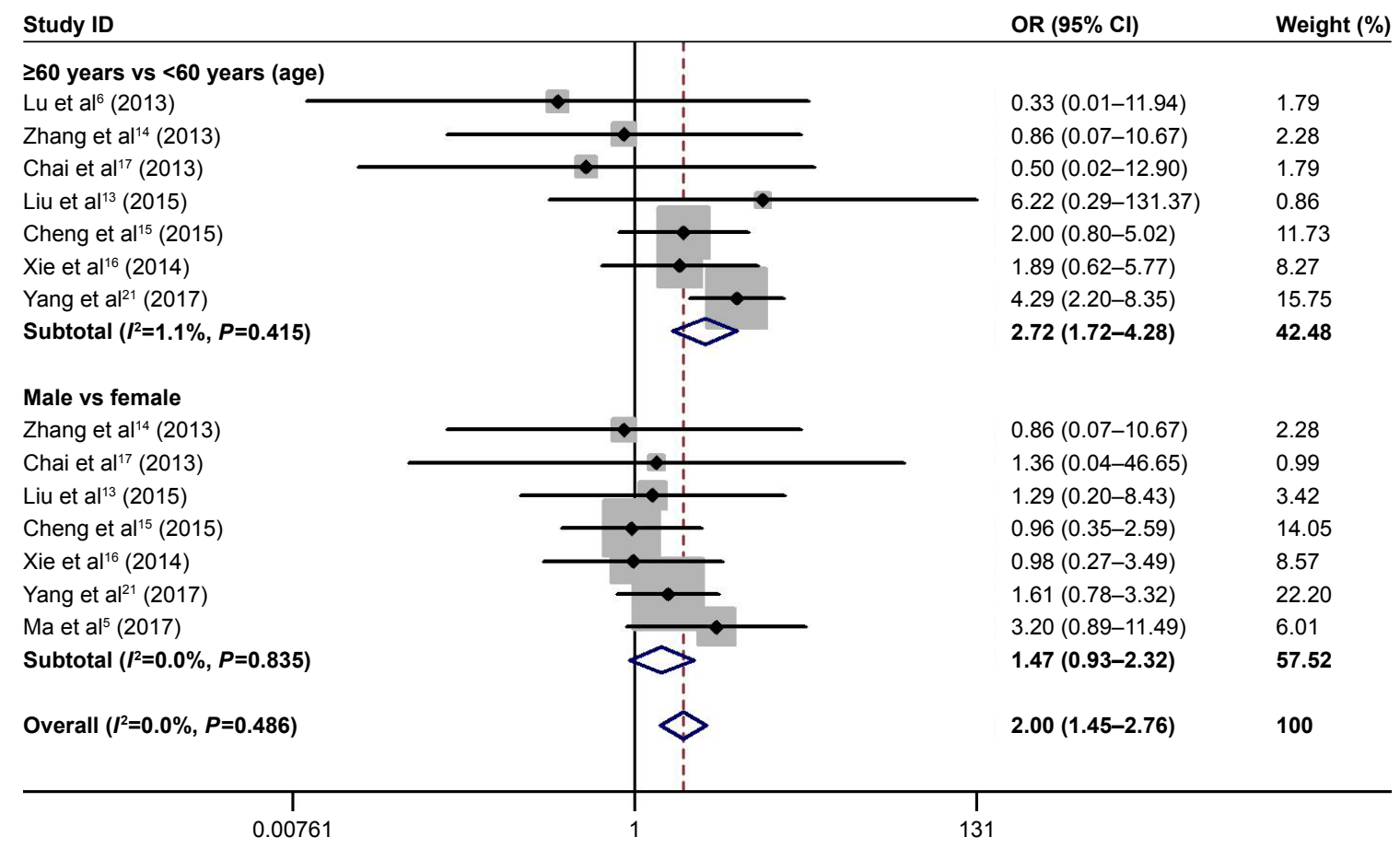

Figure 3 Forest plot on estimates of combined effects of UMCs with age or gender in H7N9 patients. Abbreviation: UMC, underlying medical condition. 
Table 2 Summary of heterogeneity and publication bias on each meta-analysis of UMCs associated with fatalities of H7N9-infected cases

\begin{tabular}{|c|c|c|c|c|c|c|}
\hline \multirow[t]{2}{*}{ Study } & \multirow{2}{*}{$\begin{array}{l}\text { Numbers } \\
\text { of studies }\end{array}$} & \multicolumn{3}{|c|}{ Heterogeneity } & \multicolumn{2}{|c|}{ Publication bias } \\
\hline & & $\mathbf{Q}$ & $P$-value & $I^{2}(\%)$ & $\begin{array}{l}\text { Egger's test } \\
{[\mathrm{t}(P)]}\end{array}$ & $\begin{array}{l}\text { Begg's test } \\
{[z(P)]}\end{array}$ \\
\hline The pooled estimate certain UMC types & 15 & 7.61 & 0.909 & 0.0 & $0.20(0.848)$ & $0.74(0.488)$ \\
\hline Having two or more UMCs & 4 & 0.52 & 0.915 & 0.0 & $1.26(0.333)$ & $0.34(0.734)$ \\
\hline CRD & 7 & 9.35 & 0.155 & 35.8 & $1.32(0.245)$ & $0.00(1.000)$ \\
\hline $\mathrm{CHD}$ & 5 & 1.01 & 0.909 & 0.0 & $0.09(0.937)$ & $0.24(0.836)$ \\
\hline CMD & 7 & 1.81 & 0.936 & 0.0 & $\mathrm{I} .43(0.213)$ & $0.60(0.548)$ \\
\hline CLD & 7 & 2.39 & $0.88 \mathrm{I}$ & 0.0 & $0.38(0.720)$ & $0.60(0.548)$ \\
\hline CCD & 8 & 1.58 & 0.979 & 0.0 & $0.35(0.74 I)$ & $0.12(0.902)$ \\
\hline $\mathrm{RD}$ & 4 & 0.11 & 0.991 & 0.0 & $-1.32(0.317)$ & $-0.34(1.000)$ \\
\hline \multicolumn{7}{|c|}{ Estimates by adjusting for the effects of age and gender } \\
\hline \multicolumn{7}{|l|}{ Age } \\
\hline 60 years of age and older & 7 & 0.59 & 0.997 & 0.0 & $0.22(0.507)$ & $0.00(1.000)$ \\
\hline $0-59$ years old & 7 & 3.42 & 0.490 & 10.5 & $0.42(0.730)$ & $0.24(0.806)$ \\
\hline \multicolumn{7}{|l|}{ Gender } \\
\hline Male & 7 & 0.74 & 0.994 & 0.0 & $-0.49(0.132)$ & $0.60(0.548)$ \\
\hline Female & 7 & 1.96 & 0.926 & 0.0 & $-0.21(0.753)$ & $0.15(0.881)$ \\
\hline \multicolumn{7}{|c|}{ The estimate of combined effects of UMCs with age or gender } \\
\hline 60 years of age and older vs $0-59$ years old & 7 & 6.07 & $0.4 \mid 4$ & 1.1 & $-2.04(0.097)$ & $1.80(0.072)$ \\
\hline Male vs female & 7 & 2.79 & 0.835 & 0.0 & $-0.22(0.750)$ & $0.30(0.764)$ \\
\hline
\end{tabular}

Abbreviations: CCD, chronic cardiovascular diseases; CHD, coronary heart disease; CLD, chronic liver disease; CMD, chronic metabolic diseases; CRD, chronic respiratory diseases; RD, rheumatic diseases; UMCs, underlying medical conditions.

0-59 years old. One can, therefore, hypothesize that UMCs enhance the outcome severity in elderly H7N9 patients. ${ }^{29}$ Contrarily, it may be that they have a good prognosis in younger H7N9 patients with UMCs. In addition, another explanation may be that the severity of UMCs is lower in
H7N9 patients 0-59 years old, compared to H7N9 patients who were 60 years and older. Our meta-analysis also found that with an increased OR, there was a significant association with increased fatality rates among female or male H7N9 patients with UMCs compared to female or male
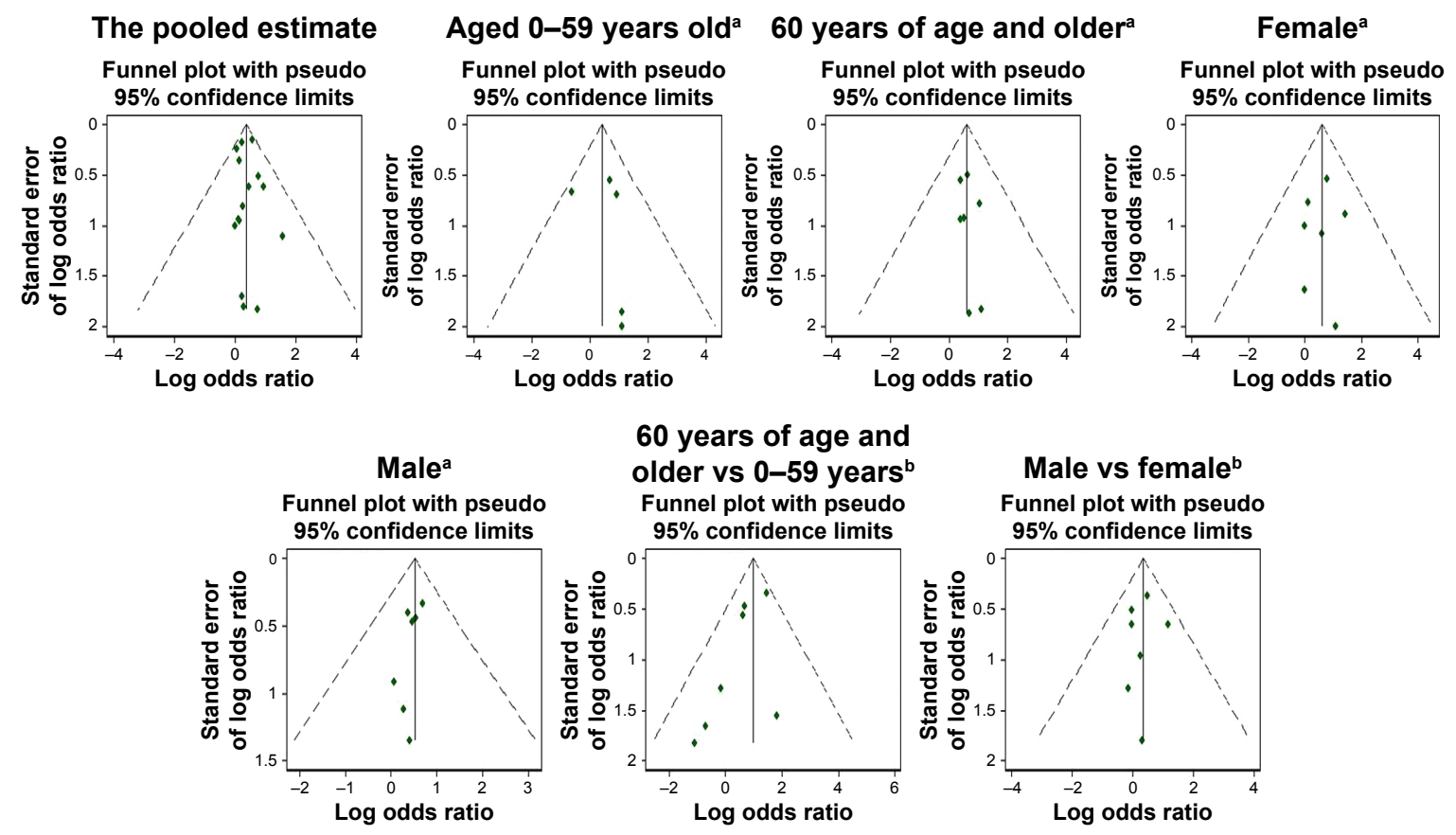

Figure 4 Funnel plots on meta-analyses of underlying medical conditions associated with fatalities of H7N9-infected cases.

Notes: a'Estimates by adjusting for the effects of age and gender. ${ }^{\mathrm{b}}$ The estimate of combined effects of UMCs with age or gender.

Abbreviation: UMC, underlying medical condition. 
H7N9 patients without UMCs. Similarly, previous studies reported that UMCs are associated with increased fatality among H7N9-infected patients as a broader group. ${ }^{5,21}$ It may help policymakers recognize and understand the gender problem in H7N9-infected patients and make appropriate and necessary intervention.

Our analysis is the first to examine the combined effects of UMCs with other potential impact factors (such as age and gender) on fatality rates among H7N9 patients. Most notably, we used a stratified comparison to examine H7N9 cases with UMCs. We found that among H7N9 patients with UMCs, the odds of death were approximately three times as high in patients aged 60 years and older than those aged $0-59$. This result is consistent with a report by Ji et al. ${ }^{7}$ Similarly, previous reports on seasonal influenzas have found that older age and preexisting concurrent chronic diseases are associated with increased fatalities from high and low pathogenic avian influenza. ${ }^{30,31}$ However, while gender is often believed to be a risk factor for severe complications and death from
H7N9 infection, ${ }^{28}$ our analysis found no significant differences between fatality rates of female and male among H7N9 patients with UMCs, which indicates that gender differences have no obvious link to H7N9 fatalities with UMCs. This may reflect the confounding effect of UMCs between gender and death in H7N9-infected patients. Nonetheless, a study with a larger sample size is needed for confirmation.

\section{Limitations}

Finally, we should note that our meta-analysis had some limitations. First, ascertainment of UMCs was mostly based on passive reporting by family members, and underreporting may have occurred because of poor recognition. Second, we did not have data on the frequency or intensity of exposure, which would affect the amount of virus involved in infection. Third, data published in a small number of reports used in the present meta-analysis were extracted from nonstandardized medical records. Fourth, there was a possible overlapping across part of the patient samples used in our study

A

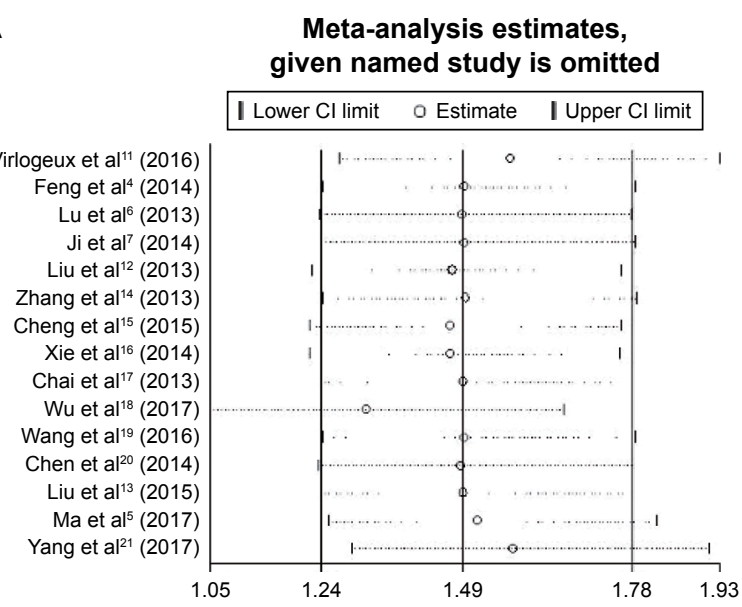

B
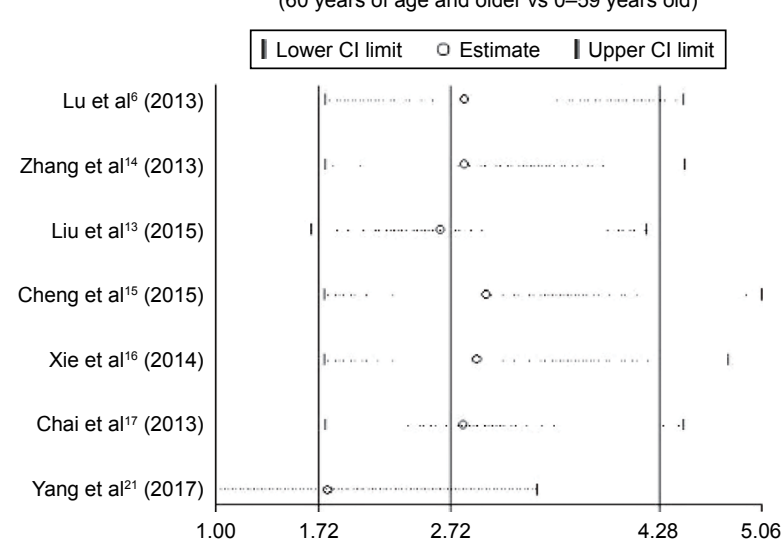

Meta-analysis estimates, given named study is omitted
(60 years of age and older vs $0-59$ years old) Lower Cl limit a Estimate I Upper Cl limit

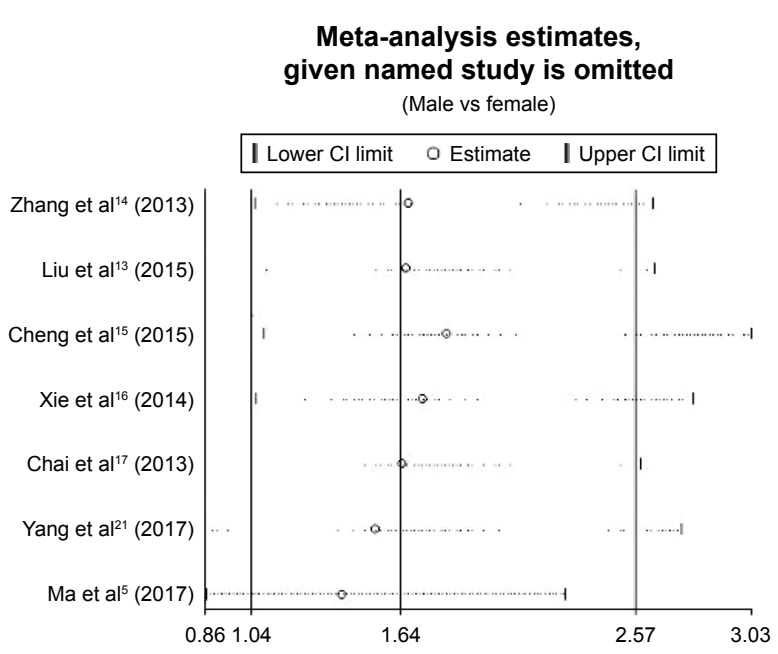

Figure 5 (Continued) 


\section{C}

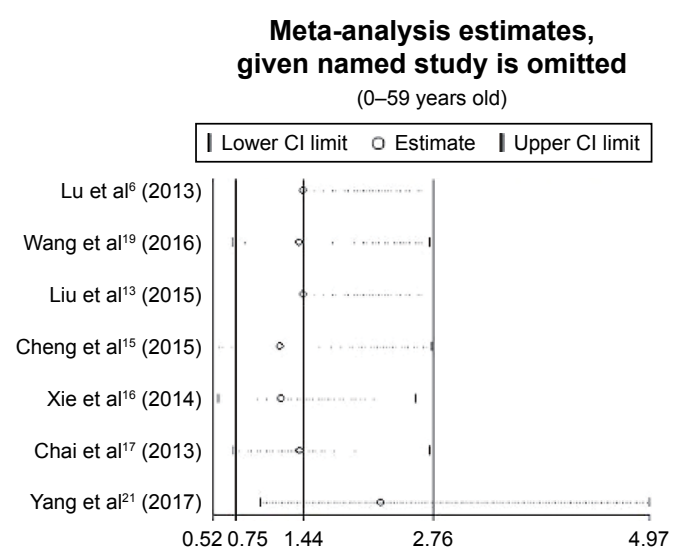

Meta-analysis estimates, given named study is omitted (Female)

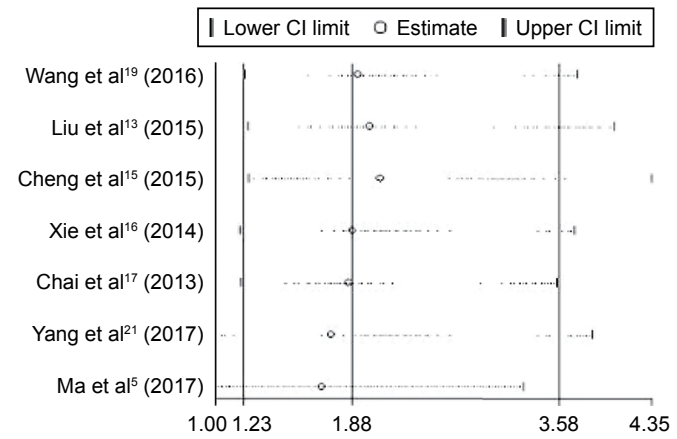

Meta-analysis estimates, given named study is omitted (60 years of age and older)

I Lower Cl limit a Estimate I Upper Cl limit

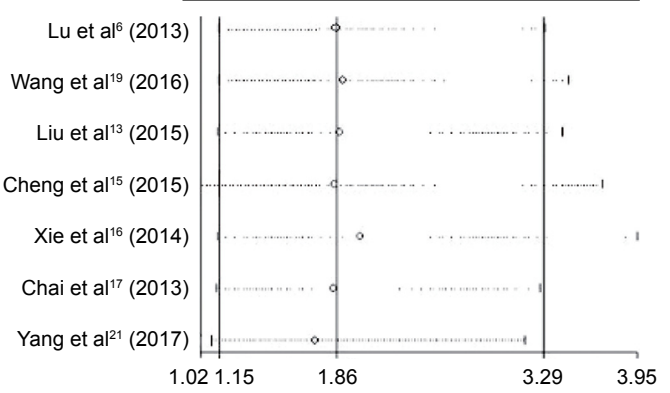

Meta-analysis estimates, given named study is omitted (Male)

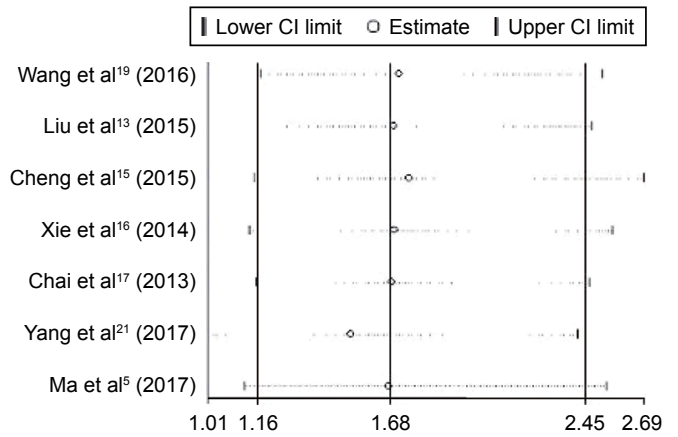

Figure 5 Sensitivity analysis plots on meta-analyses of underlying medical conditions associated with fatalities of H7N9-infected cases. Notes: (A) The pooled estimate. (B) The estimate of combined effects of underlying medical conditions with age or gender. (C) The estimates by adjusting for the effects of age and gender.

(eg, References "5" and "7", References "15" and "16", and References "19" and " 21 "). To minimize the possible overlapping, we have eliminated some repeated studies in the study inclusion/exclusion process (Figure 1). In addition, authors were contacted when the patient sample was a possible overlapping. Moreover, subgroup and stratification analyses were conducted to reduce the impact on the results from the overlapping of the patient samples. However, we still could not entirely exclude the overlapping of the patient samples. Finally, as an inherent limitation of all meta-analyses of observational data, we cannot exclude the possibility that the association is due to confounding factors. Indeed, we were not able to adjust for medication effects such as the interaction between age and gender, because these were not recorded systematically and we did not have access to individual participant data.

\section{Conclusion}

Our study was unique in adjusting for the effects of age or gender as well as UMCs and we found that UMCs were associated with higher fatality rates only among older H7N9 patients (60 years or older). Among this older population, multiple comorbid UMC, CRD, and CCD were significantly correlated with higher fatality rates compared to patients in the same age group without these UMCs. Our findings suggest that early diagnosis and treatment of CRD, CCD, and multiple comorbid UMCs may more effectively reduce fatalities and disease burden among elderly H7N9-infected patients.

Our analysis is also the first to examine the combined effects of UMCs with other potential impact factors (such as age and gender) on fatality rates among H7N9 patients. We found that the combined effects of UMCs with 60 years of age and older were significantly associated with fatalities in H7N9 patients. However, this analysis did not find a significant difference in gender comparisons. Impacts of age are substantial and significant at the risk of UMCs and death in H7N9 patients. Efforts should particularly focus on reducing fatality rates in patients with combined risks from UMCs and other significant impact factors such as age. 


\section{Acknowledgments}

We would like to thank $\mathrm{C}$ Chai, S Liu, and $\mathrm{H}$ Wang for providing additional data. This work was supported by the Hangzhou Science and Technology Project with Innovation (grant number 20131813A07).

\section{Author contributions}

Study design, first draft of manuscript, literature review, data analysis and interpretation, and critical revision: QC. Statistical analysis, data analysis and interpretation, and critical revision of text: XW. Literature review, data analysis and interpretation, and critical revision of text: GZ, LX. All authors contributed to data analysis, drafting and revising the article, gave final approval of the version to be published, and agree to be accountable for all aspects of the work.

\section{Disclosure}

The authors report no conflicts of interest in this work.

\section{References}

1. Zhang F, Bi Y, Wang J, et al. Human infections with recently-emerging highly pathogenic H7N9 avian influenza virus in China. $J$ Infect. 2017;75(1):71-75.

2. Arima Y, Vong S. World Health Organization Outbreak Response Team. Human infections with avian influenza A (H7N9) virus in China: preliminary assessments of the age and sex distribution. Western Pac Surveill Response J. 2013;4(2):1-3.

3. Zhang YM, Liu JM, Yu L, et al. Prevalence and characteristics of hypoxic hepatitis in the largest single-centre cohort of avian influenza A (H7N9) virus-infected patients with severe liver impairment in the intensive care unit. Emerg Microbes Infect. 2016;5(1):e1.

4. Feng F, Jiang Y, Yuan M, et al. Association of radiologic findings with mortality in patients with avian influenza H7N9 pneumonia. PLoS One. 2014;9(4):e93885.

5. Ma W, Huang H, Chen J, et al. Predictors for fatal human infections with avian H7N9 influenza, evidence from four epidemic waves in Jiangsu Province, Eastern China, 2013-2016. Influenza Other Respir Viruses. 2017;11:418-424.

6. Lu S, Zheng Y, Li T, et al. Clinical findings for early human cases of influenza A (H7N9) virus infection, Shanghai, China. Emerg Infect Dis. 2013;19(7):1142-1146.

7. Ji H, Gu Q, Chen LL, et al. Epidemiological and clinical characteristics and risk factors for death of patients with avian influenza A H7N9 virus infection from Jiangsu Province, Eastern China. PLoS One. 2014; 9(3):e89581

8. Huang F, Guo J, Zou Z, et al. Angiotensin II plasma levels are linked to disease severity and predict fatal outcomes in H7N9-infected patients. Nat Commun. 2014;5:3595.

9. Wells GA, Shea B, O'Connell D, et al. The Newcastle-Ottawa Scale (NOS) for Assessing the Quality of Nonrandomised Studies in the Meta-Analysis; 2017. Available from: http://www.ohri.ca/programs/ clinical_epidemiology/oxford.htm. Accessed December 7, 2017.

10. Cho H, Kim H, Na D, et al. Meta-analysis method for discovering reliable biomarkers by integrating statistical and biological approaches: an application to liver toxicity. Biochem Biophys Res Commun. 2016; 471(2):274-281

11. Virlogeux V, Yang J, Fang VJ, et al. Association between the severity of influenza A (H7N9) virus infections and length of the incubation period. PLoS One. 2016;11(2):e0148506.
12. Liu S, Sun J, Cai J, et al. Epidemiological, clinical and viral characteristics of fatal cases of human avian influenza A (H7N9) virus in Zhejiang Province, China. J Infect. 2013;67(6):595-605.

13. Liu X, Zhang Y, Xu X, et al. Evaluation of plasma exchange and continuous veno-venous hemofiltration for the treatment of severe avian influenza A (H7N9): a cohort study. Ther Apher Dial. 2015;19(2): $178-184$.

14. Zhang A, Huang Y, Tian D, et al. Kinetics of serological responses in influenza A (H7N9)-infected patients correlate with clinical outcome in China, 2013. Euro Surveill. 2013;18(50):20657.

15. Cheng QL, Ding H, Sun Z, et al. Retrospective study of risk factors for mortality in human avian influenza A (H7N9) cases in Zhejiang Province, China, March 2013 to June 2014. Int J Infect Dis. 2015;39:95-101.

16. Xie L, Cheng QL, Ding H, et al. Retrospective study of risk factors of mortality in human avian influenza A (H7N9) cases in Hangzhou. Chin J Infect Dis. 2014;32(11):683-687.

17. Chai CL, Chen EF, Chen ZP, et al. Clinical and epidemiological characteristics of the 6 confirmed human cases with novel influenza A (H7N9) virus infection in Zhejiang. Zhonghua Liu Xing Bing Xue Za Zhi. 2013;34(5):443-445.

18. Wu ZQ, Zhang Y, Zhao N, et al. Comparative epidemiology of human fatal infections with novel, high (H5N6 and H5N1) and low (H7N9 and $\mathrm{H} 9 \mathrm{~N} 2)$ pathogenicity avian influenza A viruses. Int J Environ Res Public Health. 2017;14(3):E263.

19. Wang H, Xiao XC, Lu J, et al. Factors associated with clinical outcome in 25 patients with avian influenza A (H7N9) infection in Guangzhou, China. BMC Infect Dis. 2016;16(1):534.

20. Chen Y, Li X, Tian L, et al. Dynamic behavior of lymphocyte subgroups correlates with clinical outcomes in human H7N9 infection. J Infect. 2014;69(4):358-365.

21. Yang Y, Zhong H, Song T, et al. Epidemiological and clinical characteristics of humans with avian influenza A (H7N9) infection in Guangdong, China, 2013-2017. Int J Infect Dis. 2017;65:148-155.

22. Pebody RG, Mclean E, Zhao H, et al. Pandemic influenza A (H1N1) 2009 and mortality in the United Kingdom: risk factors for death, April 2009 to March 2010. Euro Surveill. 2010;15(20):19571.

23. Ribeiro AF, Pellini ACG, Kitagawa BY, et al. Risk factors for death from influenza A (H1N1)pdm09, State of São Paulo, Brazil, 2009. PLoS One. 2015;10(3):e0118772.

24. Hennessy TW, Bruden D, Castrodale L, et al. A case-control study of risk factors for death from 2009 pandemic influenza A (H1N1): is American Indian racial status an independent risk factor? Epidemiol Infect. 2016;144(2):315-324.

25. Guan Y, Farooqui A, Zhu H, Dong W, Wang J, Kelvin DJ. H7N9 incident, immune status, the elderly and a warning of an influenza pandemic. J Infect Dev Ctries. 2013;7(4):302-307.

26. Bermejo-Martin JF, Almansa R, Ortiz de Lejarazu R. Weakened immunity in aged hosts with comorbidities as a risk factor for the emergence of influenza A H7N9 mutants. J Infect Dev Ctries. 2013;7(6):497-498.

27. Gao HN, Lu HZ, Cao B, et al. Clinical findings in 111 cases of influenza A (H7N9) virus infection. N Engl J Med. 2013;368(24):2277-2285.

28. Dudley JP, Mackay IM. Age-specific and sex-specific morbidity and mortality from avian influenza A (H7N9). J Clin Virol. 2013;58(3): 568-570.

29. Qiu C, Fratiglioni L. A major role for cardiovascular burden in agerelated cognitive decline. Nat Rev Cardiol. 2015;12(5):267-277.

30. Simonsen L, Taylor RJ, Young-Xu Y, Haber M, May L, Klugman KP. Impact of pneumococcal conjugate vaccination of infants on pneumonia and influenza hospitalization and mortality in all age groups in the United States. MBio. 2011;2(1):e00309-e00310.

31. Wu P, Peng Z, Fang VJ, et al. Human infection with influenza A (H7N9) virus during 3 major epidemic waves, China, 2013-2015. Emerg Infect Dis. 2016;22(6):964-972. 


\section{Publish your work in this journal}

Therapeutics and Clinical Risk Management is an international, peerreviewed journal of clinical therapeutics and risk management, focusing on concise rapid reporting of clinical studies in all therapeutic areas, outcomes, safety, and programs for the effective, safe, and sustained use of medicines. This journal is indexed on PubMed Central, CAS,

EMBase, Scopus and the Elsevier Bibliographic databases. The manuscript management system is completely online and includes a very quick and fair peer-review system, which is all easy to use. Visit http://www.dovepress.com/testimonials.php to read real quotes from published authors.

Submit your manuscript here: http://www.dovepress.com/therapeutics-and-clinical-risk-management-journal 